\title{
Sergentomyia (Rondanomyia) ingrami Newstead, 1914, Sergentomyia (Rondanomyia) dureni Parrot, 1934 et Sergentomyia bamoni Abonnenc, 1958
}

(Diptera, Phlebotomidae).

\section{Etude morphologique des stades pré-imaginaux et notes bio-écologiques}

\author{
par J. TROUILLET
}

Collaboration technique: J.-M. Boudzoumou, pour les captures

A.-S. Bimangou, pour les montages.

Département de Biologie animale, Equipe de Bio-écologie des Vecteurs, Faculté des Sciences, B.P. 69, Brazzaville, République populaire du Congo.

\section{Résumé.}

L'auteur a obtenu en élevage les stades pré-imaginaux de $S$. ingrami, $S$. dureni et $S$. hamoni. Il fait l'étude morphologique de ceux-ci et précise la durée du développement post-embryonnaire. La chétotaxie des larves du $4^{\circ}$ stade de $S$. hamoni présente des particularités par rapport aux autres espèces décrites.

\section{Summary:}

Sergentomyia (Rondanomyia) ingrami Newstead, 1914, Sergentomyia (Rondanomyia) dureni Parrot, 1934 and Sergentomyia hamoni Abonnenc, 1958 (Diptera Phlebotomidae). Morphology of immature stages and biocological accounts.

Immature stages of $S$. ingrami, $S$. dureni and $S$. hamoni have been obtained in laboratory culture. The morphological characters of egg, larva and pupa are studied, the duration of post-embryonic development is specified. The chaetotaxy of fourth-instar larvae of $S$. hamoni is distinguishable from the other species described.

Accepté le 3 janvier 1979. 
Dans le cadre de nos recherches sur la biologie des Phlébotomes congolais, les formes pré-imaginales de Grassomyia ghesquierei Parrot, 1929, Sergentomyia (Sergentomyia) bedfordi firmatus Parrot et Malbrant, 1945 et Sergentomyia (Sergentomyia) bedfordi medius Kirk et Lewis, 1950 ont déjà été décrites (Trouillet, 1976 et 1977). Poursuivant la mise en élevage de Phlébotomes sauvages, nous avons récemment observé le développement post-embryonnaire de Sergentomyia ingrami, S. dureni et S. hamoni.

L'étude morphologique des stades pré-imaginaux de ces trois espèces sera suivie de quelques remarques sur leur cycle et leur écologie.

\section{I. - Lieux, matériels et méthodes}

Toutes les femelles mises en élevage ont été capturées dans la région de YakaYaka $\left(04^{\circ} 22^{\prime}\right.$ Sud, $15^{\circ} 09^{\prime}$ Est), village situé à $21 \mathrm{~km}$ au Sud-Ouest de Brazzaville (Trouillet et al., sous presse). La capture s'est effectuée à l'aide d'un simple tube dont on recouvre l'Insecte au repos.

Les deux femelles de $S$. dureni étaient posées, l'une sur le mur d'une case habitée du village de Yaka-Yaka, l'autre sur le tronc d'un arbre coupé au bord de la rivière Maloto; la femelle de $S$. ingrami et les femelles de $S$. hamoni, sur des troncs d'arbres dans la forêt-galerie qui borde la rivière Djoumouna.

Ces femelles et leurs pontes ont été conservées suivant la méthode déjà exposée (Trouillet, 1977) et les larves. nourries avec de la poudre de foie desséchée (Gemetchu, 1971 ; Killick-Kendrick et al., 1977).

\section{II. - Etude morphologique}

Le montage des œufs, des larves et des nymphes a été réalisé au baume du Canada après éclaircissement dans une solution de potasse à $20 \%$, mordançage dans le liquide de Berlèse, puis déshydratation dans l'alcool à $70^{\circ}$, dans l'alcool à $95^{\circ}$ et dans la créosote de hêtre.

La chétotaxie des larves est étudiée selon la nomenclature numérique d'Abonnenc (1956).

\section{Sergentomyia ingrami.}

1. - L'éuF (fig. 1).

Il mesure 0,35 $\mathrm{mm}$ de longueur sur 0,12 $\mathrm{mm}$ de largeur. Comme chez G. ghesquierei, $S$. bedfordi firmatus et $S$. bedfordi medius, les granulations de l'exochorion figurent des cellules polygonales irrégulières. 


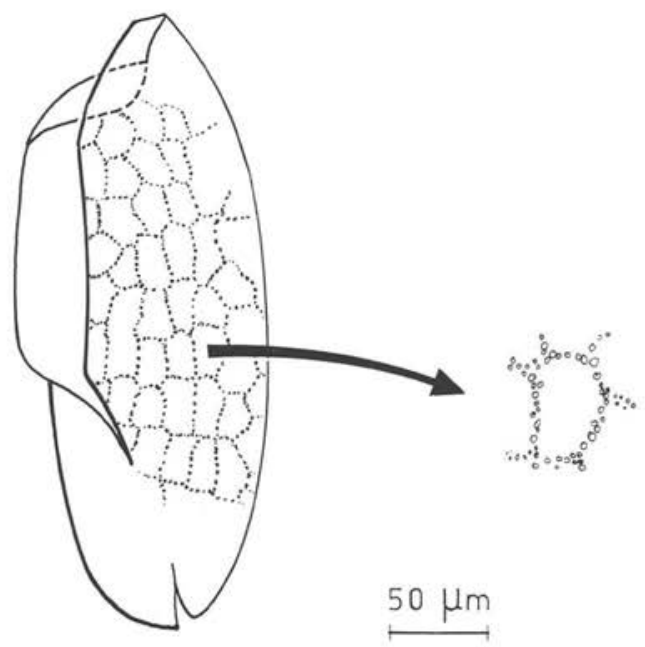

Fig. 1. L'œuf de S. ingrami.

2. - La larve du $4^{e} \operatorname{stade}(f i g .2, A$ et $B$ et tableau $I$ ).

Nous avons mesuré 5 larves et obtenu les dimensions moyennes suivantes:

- longueur totale : $2,50 \mathrm{~mm}(2,29-2,80)\left({ }^{*}\right)$;

- dimensions de la tête : $0,38 \mathrm{~mm}$ de longueur $(0,36-0,40)$ sur $0,25 \mathrm{~mm}$ de largeur $(0,23-0,26)$;

- longueur des soies caudales internes: $1,93 \mathrm{~mm}(1,90-2,00)$;

- longueur des soies caudales externes : $1,82 \mathrm{~mm}(1,75-1,85)$.

La longueur des soies caudales apparaît difficile à employer comme caractère taxonomique. Ces soies sont, en effet, bien souvent cassées, tire-bouchonnées ou contournées.

\subsection{La tête (fig. 3).}

Les soies 1, 2, 4, 5, 6 sont lisses. Les soies 3 barbulées. Les soies 7, 8, 9, épineuses (les soies 7 et 8 étant subégales et la soie 9 un peu plus courte que les deux autres).

\subsection{Le thorax.}

Prothorax antérieur (fig. 3).

Région dorsale. Soies 1, 2, 3 épineuses. Soie $3(120 \mu \mathrm{m})$ un peu plus grande que la soie $1(109 \mu \mathrm{m})$. La soie 2 est petite $(36 \mu \mathrm{m})$.

(*) La moyenne, suivie entre parenthèses, des valeurs extrêmes. 

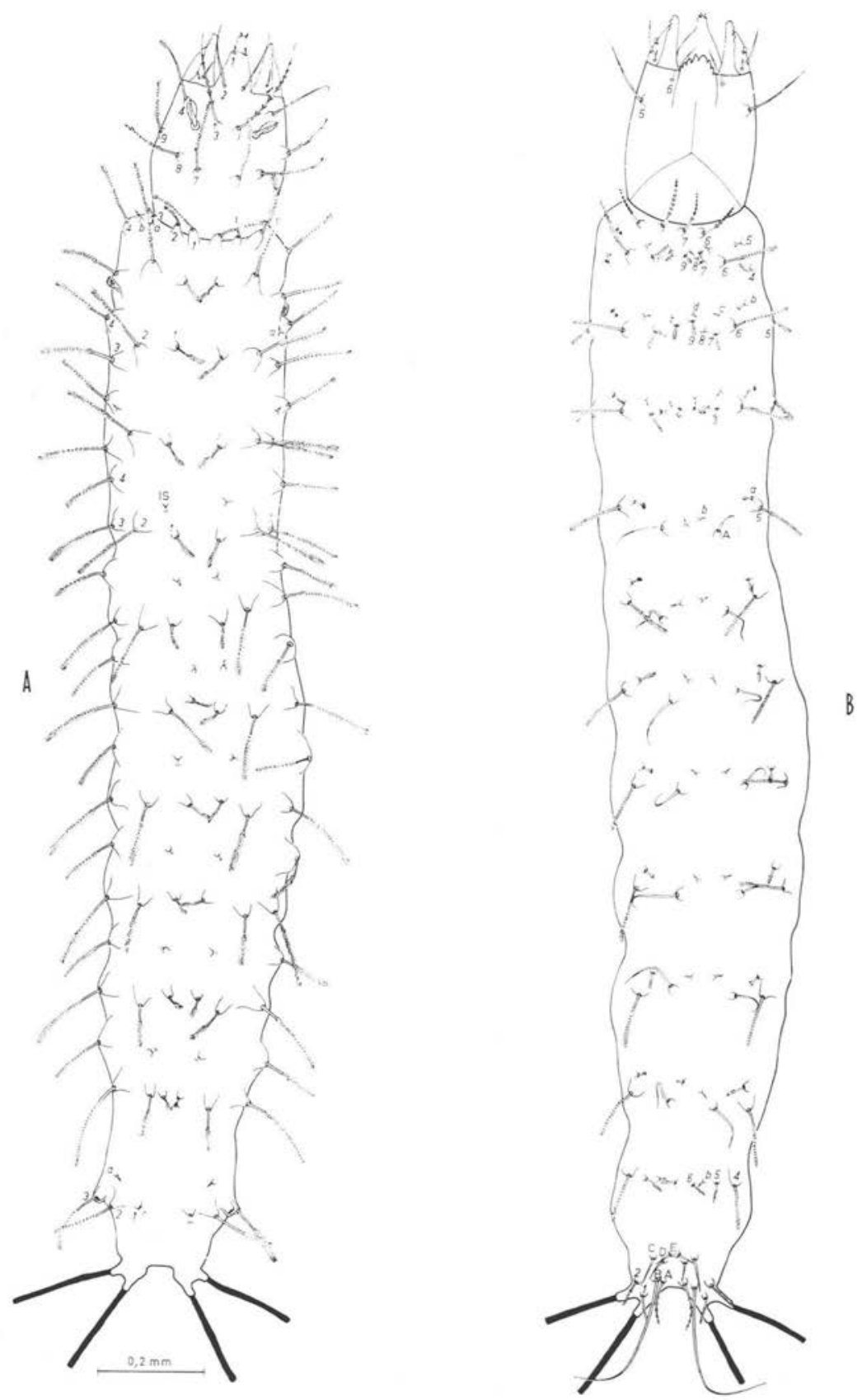

Fig. 2. S. ingrami. Larve du $4^{\text {e }}$ stade. A: vue dorsale; B: vue ventrale. 


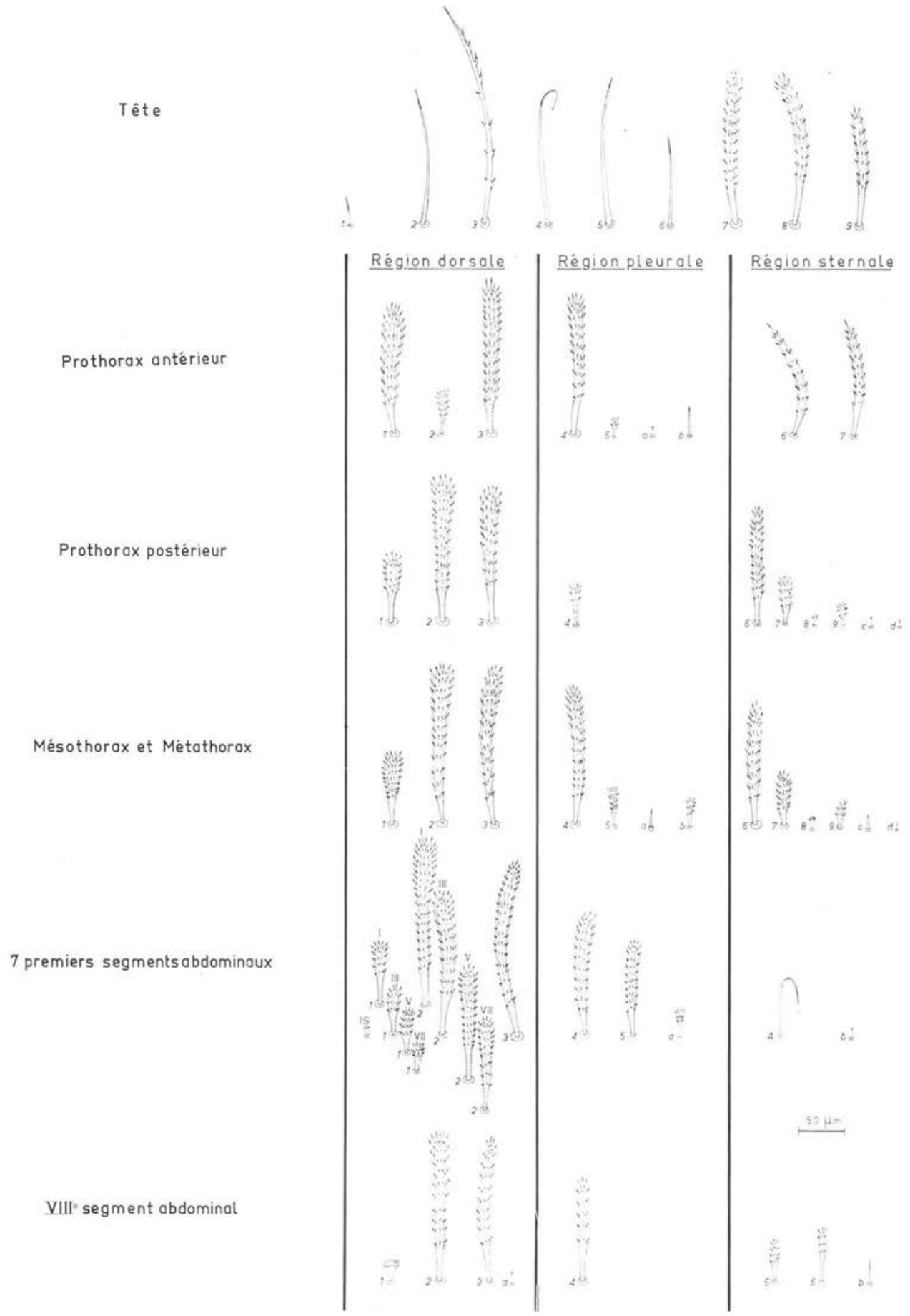

Fig. 3. S. ingrami. Soies de la larve du $4^{e}$ stade. 
Région pleurale. Les microsoies $a$ et $b$ sont présentes, simples et pointues, $a$ étant nettement plus courte que $b$. La soie 4 de taille comparable à la soie 3 , est longue et épineuse. La soie 5 , courte $(14 \mu \mathrm{m})$, porte 5 ou 6 épines terminales.

Région sternale. Soies 6 et 7, subégales, densément barbulées.

Prothorax postérieur (fig. 3).

Région dorsale. Soies $1,2,3$ épineuses. Seule la soie 3 conserve à peu près la même taille que sur le prothorax antérieur; la soie 1 est plus petite $(52 \mu \mathrm{m})$, la soie 2 plus grande $(124 \mu \mathrm{m})$.

Région pleurale. Elle porte les stigmates respiratoires antérieurs et une paire de soies 4 , petites $(35 \mu \mathrm{m})$ et épineuses.

Région sternale. Soies $6(107 \mu \mathrm{m}), 7(42 \mu \mathrm{m}), 8(10 \mu \mathrm{m}), 9(19 \mu \mathrm{m})$ épineuses. La petite soie 8 est terminée par 3 ou 4 épines. Microsoies $c$ et $d$ courtes et pointues.

Mésothorax et métathorax (fig. 3).

Région dorsale. Soies $1,2,3$ épineuses et de tailles légèrement supérieures à leurs homologues du prothorax postérieur.

Région pleurale. Soie 4 et soie 5 épineuses, microsoie $a$ simple et pointue, microsoie $b$ bien développée $(19-20 \mu \mathrm{m})$ et barbulée.

Région sternale. Toutes les soies sont semblables à celles de la région homologue du prothorax postérieur.

\subsection{L'abdomen.}

Les sept premiers segments abdominaux (fig. 3).

Région dorsale. Les trois paires de soies $(1,2,3)$ sont épineuses. Seules les soies 3 ont une taille constante sur tous les segments. Les soies 1 et 2 décroissent régulièrement du $l^{\text {er }}$ au $\mathrm{VII}^{\circ}$ segment; sur les segments I, III, V et VII les dimensions sont respectivement les suivantes:

- soies 1: $47 \mu \mathrm{m}, 38 \mu \mathrm{m}, 32 \mu \mathrm{m}, 18 \mu \mathrm{m}$;

— soies 2: $136 \mu \mathrm{m}, 99 \mu \mathrm{m}, 80 \mu \mathrm{m}, 59 \mu \mathrm{m}$.

Les soies intersegmentaires, facilement observables, portent 5-6 épines terminales.

Région pleurale. Deux paires de soies $(4,5)$ épineuses, subégales sur tous les segments. Microsoies $a$ bien développées (20-24 $\mu \mathrm{m})$ et barbulées.

Région sternale. Soies A longues et lisses, généralement recourbées à l'extrémité. Microsoies $b$ courtes et pointues.

Le VIII segment abdominal (fig. 3).

Région dorsale. Trois paires de soies $(1,2,3)$ épineuses; les soies 1 (14 $\mu \mathrm{m})$ étant encore un peu plus réduites que sur le $\mathrm{VII}^{e}$ segment, les soies 2 et 3 bien développées et de mêmes dimensions. Microsoies $a$ simples, situées à côté des stigmates respiratoires postérieurs. 


\begin{tabular}{|c|c|c|c|c|c|c|c|c|c|c|c|c|c|c|c|c|c|c|c|}
\hline$\underline{x}$ & 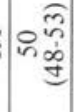 & 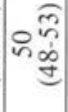 & 1 & 1 & 1 & 1 & 1 & 1 & 1 & 1 & 1 & 1 & 1 & 1 & $\infty \frac{\widehat{a}}{5}$ & 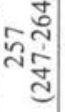 & 6 & & \\
\hline$\Xi$ & $\underset{I}{\simeq}$ & 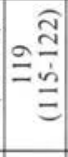 & 고엉 & 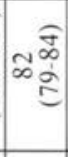 & ஓे & 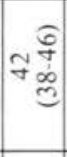 & 1 & I & 1 & 1 & $=\frac{\widehat{\theta}}{0}$ & $\underline{\underline{\underline{n}}}$ & 1 & 1 & 1 & 1 & 1 & 1 & 1 \\
\hline 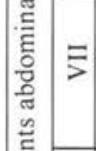 & 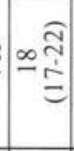 & 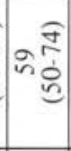 & 总 & 으을 & कू & 1 & 1 & 1 & 1 & $\sigma \frac{\hat{\sigma}}{\infty}$ & 춿 & $=\frac{\widehat{\tau}}{0}$ & 1 & 1 & 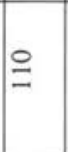 & 1 & 1 & I & 1 \\
\hline 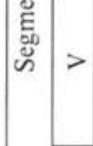 & 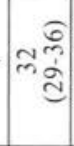 & $\approx \frac{\widehat{2}}{5}$ & 들 & 킁 & مू & 1 & 1 & 1 & 1 & $a \frac{\sigma}{\infty}$ & ฟঙิ & $=\frac{\widehat{\tau}}{0}$ & 1 & 1 & $\stackrel{ }{=}$ & 1 & 1 & 1 & 1 \\
\hline$\Xi$ & 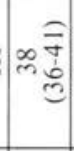 & ระ & 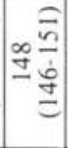 & $\stackrel{\frac{\sigma}{=}}{\circ}$ & ๙ & 1 & 1 & 1 & 1 & $a \frac{\hat{0}}{\infty}$ & 청 & $=\frac{\widehat{\simeq}}{0}$ & 1 & 1 & $\cong$ & 1 & 1 & 1 & 1 \\
\hline- & 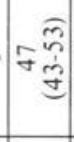 & 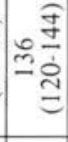 & 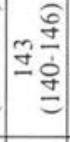 & 응 & $\approx \frac{a}{a}$ & 1 & 1 & 1 & 1 & $a \frac{0}{\infty}$ & 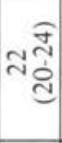 & $=\frac{\bar{\tau}}{0}$ & 1 & 1 & $\stackrel{ }{\varrho}$ & 1 & 1 & 1 & 1 \\
\hline 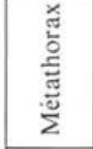 & 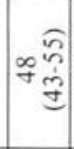 & 至 & 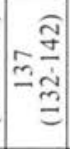 & 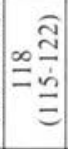 & \% & $\propto \frac{\hat{0}}{\grave{0}}$ & 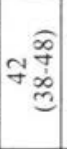 & $\therefore \frac{\hat{0}}{\infty}$ & ㅎํㅇ & $1^{\prime}$ & $=\frac{\widehat{T}}{\underline{0}}$ & ิㅗำ & ט & 0 & 1 & 1 & 1 & 1 & \\
\hline 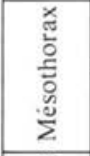 & 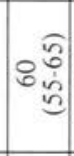 & ल⿸厂 & 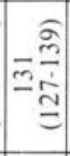 & $\stackrel{\infty}{\underline{\Xi}}$ & r. & 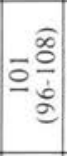 & 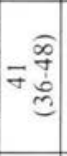 & $2 \frac{6}{5}$ & 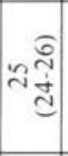 & 1 & $=\frac{\widehat{A}}{0}$ & ๙ิ) & 。로 & $0 \stackrel{\overbrace =}{\circ}$ & 1 & 1 & 1 & 1 & \\
\hline 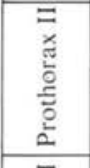 & 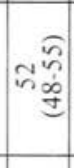 & 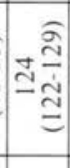 & $\stackrel{\frac{n}{2}}{\underline{0}}$ & 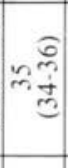 & 1 & $\stackrel{\underline{0}}{\underline{0}}$ & 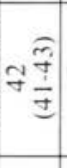 & ○文 & $\begin{array}{r}\tilde{A} \\
-\stackrel{\text { I }}{=} \\
=\end{array}$ & 1 & 1 & 1 & $0 \stackrel{?}{\tilde{g}}$ & $0 \stackrel{0}{\tilde{n}}$ & 1 & 1 & 1 & 1 & \\
\hline $\begin{array}{l}\bar{x} \\
\mathbb{w} \\
\vdots \\
\vdots \\
\vdots \\
\vdots\end{array}$ & 응 & $\underset{\substack{\infty \\
\hdashline}}{\infty}$ & 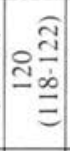 & 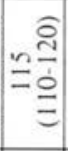 & $\pm \frac{\pi}{\check{n}}$ & 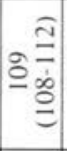 & 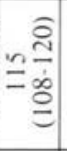 & 1 & 1 & 1 & 욜 & 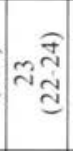 & 1 & | & 1 & 1 & 1 & 1 & \\
\hline 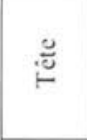 & 흘 & $\stackrel{\cong}{=}$ & 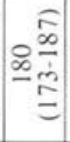 & 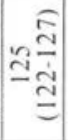 & $\underline{\infty}$ & 용 & 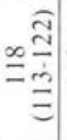 & 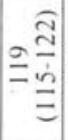 & 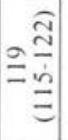 & 1 & 1 & 1 & 1 & 1 & 1 & 1 & 1 & 11 & \\
\hline is & - & $N$ & $m$ & $\nabla$ & in & 0 & r & $\infty$ & $a$ & $\simeq$ & न & 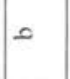 & 0 & $\sigma$ & $<$ & 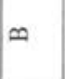 & $u$ & 0 & (1) \\
\hline
\end{tabular}




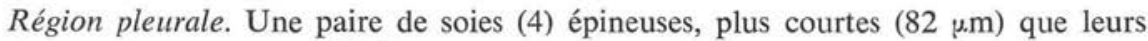
homologues des segments précédents.

Région sternale. Deux paires de soies $(5,6)$ épineuses, les soies 6 étant un peu plus grandes $(42 \mu \mathrm{m})$ que les soies $5(30 \mu \mathrm{m})$. Une paire de microsoies $b$ courtes et pointues.

\section{Le IX segment abdominal.}

Les quatre soies caudales sont longues et fortes, insérées sur deux lobes dorsaux qui portent également deux paires de soies épineuses $(1,2)$, de tailles semblables $(50 \mu \mathrm{m})$.

Au-dessous de ces lobes, se situent les soies A, B, C, D et E. Les soies A sont légèrement barbulées; toutes les autres sont lisses. Les soies $\mathrm{B}$, les plus développées, sont environ trois fois plus longues que les soies $\mathrm{A}$.

\section{3. - La larve DU $1^{\text {er }}$ STADe.}

Longueur totale : $0,87 \mathrm{~mm}(0,80-0,95)$;

Longueur des soies caudales: $0,87 \mathrm{~mm}(0,85-0,90)$;

Dimensions de la tête : $0,12 \mathrm{~mm}$ de longueur sur $0,08 \mathrm{~mm}$ de largeur.

\subsection{La tête.}

Présence du ruptor-ovi. Les soies $1,2,4,5,6$ sont lisses; les soies 3 légèrement barbulées; les soies 7, 8, 9 épineuses.

\subsection{Le thorax.}

Prothorax antérieur. Les soies 1 sont absentes; les soies 2 et 4 épineuses (les soies 2 étant les plus développées); les soies 3 lisses; les soies 5 courtes et pointues; les soies 6 et 7 légèrement barbulées.

Prothorax postérieur. Absence de stigmates respiratoires antérieurs. Les soies 1, 2, 3, 4, 7 sont épineuses, les soies 7 étant de petite taille. Les soies 6 sont assez longues et lisses; les soies 8 et 9 courtes et simples.

Mésothorax et métathorax. Les soies 1, 2, 3, 4, 6, 7, 8, 9 sont semblables à leurs homologues du prothorax postérieur. Les soies 5 sont courtes et simples.

Sur les segments thoraciques, les microsoies $a, b, c$, et $d$ sont invisibles.

\subsection{L'abdomen.}

Sur les sept premiers segments, les soies 1, 2, 3 sont épineuses (les soies 3 étant les plus développées). Les soies 4 épineuses et les soies 5 faiblement spiculées, sont subégales. Les soies A sont lisses. Le soies intersegmentaires sont présentes mais difficilement observables.

Sur le $\mathrm{VIII}^{\circ}$ segment, comme sur les segments précédents, les soies 1, 2, 3 sont épineuses (les soies 3 étant les plus développées). Les soies 4 sont barbulées ; les soies 5 et 6 courtes et lisses. Les microsoies $a$ et $b$ sont visibles alors que nous ne les avons pas vues sur les segments I à VII. Présence de la seule paire de stigmates. 
Sur le IX ${ }^{e}$ segment, les soies 1 et 2 sont épineuses, les soies 2 étant les plus développées; les soies A, B, C, D, E sont lisses.

4. - LA NYMPHE.

L'examen de 5 exuvies nymphales a permis d'observer les soies caractéristiques de ce stade (Trouillet, 1976) sans relever de caractères particuliers.

\section{Sergentomyia dureni.}

1. - L'œuF (fig. 4).

Il mesure $0,35 \mathrm{~mm}$ de longueur sur $0,11 \mathrm{~mm}$ de largeur. L'ornementation de l'exochorion est comparable à celle de $S$. ingrami.

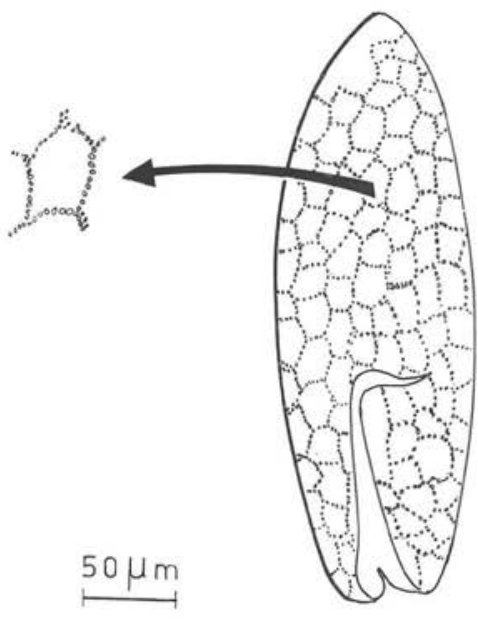

Fig. 4. L'œuf de $S$. dureni.

2. - La LARVe du $4^{\circ} \operatorname{stade}$ (fig. 5, $A$ et $B$ et tableau II).

Nous avons mesuré 5 larves et obtenu les dimensions moyennes suivantes:

- longueur totale : $2,38 \mathrm{~mm}(2,21-2,58)$;

- dimensions de la tête : $0,36 \mathrm{~mm}$ de longueur $(0,34-0,38)$ sur $0,26 \mathrm{~mm}$ de largeur $(0,25-0,27)$;

- longueur des soies caudales internes: 2,20 mm $(2,00-2,40)$;

- longueur des soies caudales externes: 1,95 mm (1,85-2,00).

2.1. La tête (fig. 6)

Les soies céphaliques sont semblables à celles de $S$. ingrami. 

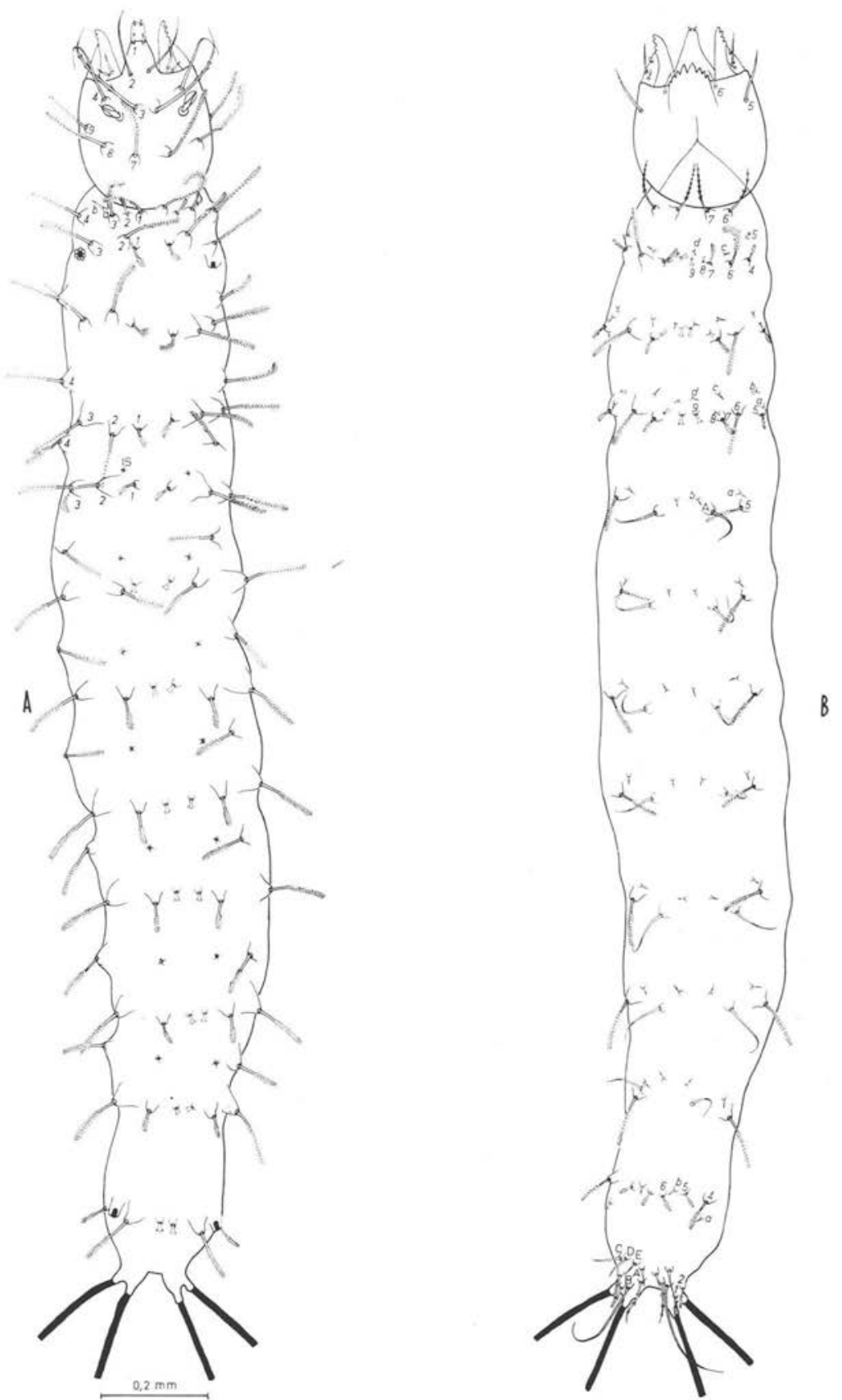

Fig. 5. S. dureni. Larve du $4^{e}$ stade. A : vue dorsale; B: vue ventrale. 


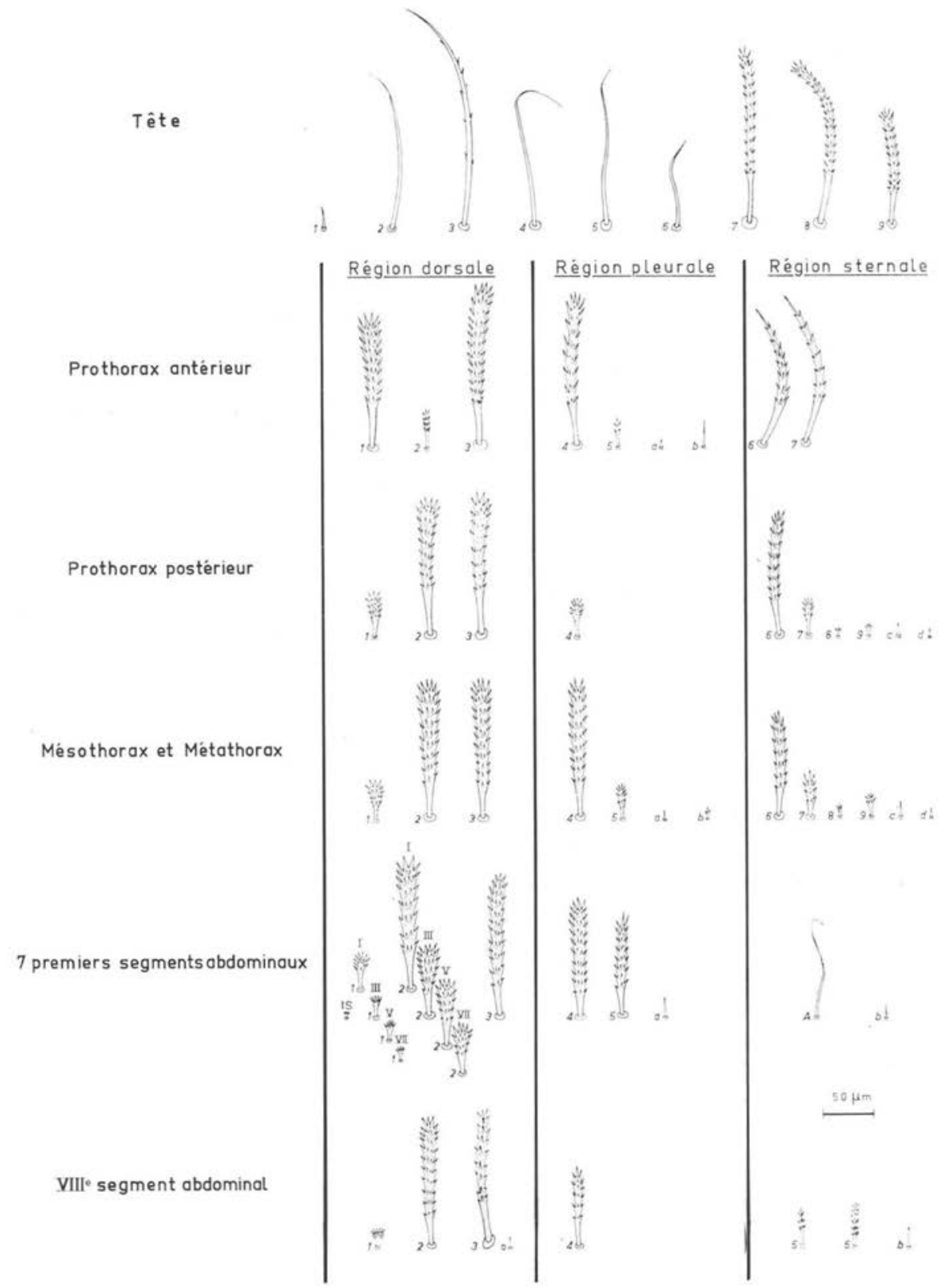

Fig. 6. S. dureni. Soies de la larve du $4^{e}$ stade. 


\begin{tabular}{|c|c|c|c|c|c|c|c|c|c|c|c|c|c|c|c|c|c|c|c|}
\hline 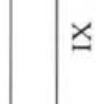 & 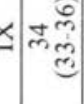 & ले & 1 & 1 & 1 & & 1 & 1 & 1 & 1 & 1 & 1 & 1 & 1 & स市 & 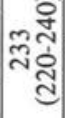 & $\stackrel{\infty}{\forall}$ & లి & ম \\
\hline 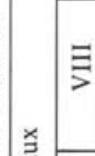 & 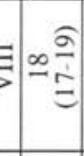 & $\approx \frac{8}{6}$ & 的 & 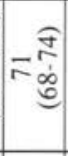 & ก & 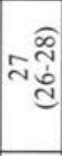 & 1 & I & I & 1 & $\circ \frac{\widehat{\simeq}}{\infty}$ & $\cong \frac{E}{ \pm}$ & 1 & 1 & 1 & 1 & 1 & 1 & 1 \\
\hline 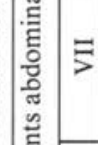 & $= \pm \frac{\sigma}{\grave{c}}$ & †京 & 응 & ஃล & $\infty \frac{\widehat{\alpha}}{\infty}$ & 1 & 1 & 1 & 1 & $n$ & $\approx \frac{\widehat{\sigma}}{\beth}$ & $=\frac{\widehat{\imath}}{0}$ & 1 & 1 & $\stackrel{ }{=}$ & 1 & 1 & 1 & 1 \\
\hline 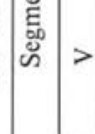 & 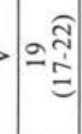 & 㛡 & 웜 & ๙ลำ & ने & 1 & 1 & 1 & 1 & $n$ & 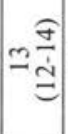 & $=\frac{\hat{\theta}}{0}$ & 1 & 1 & $\stackrel{ }{=}$ & 1 & 1 & 1 & I \\
\hline$\Xi$ & 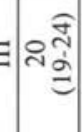 & 웅 & 웡 & 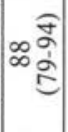 & ळ & 1 & 1 & 1 & 1 & $n$ & 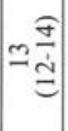 & $=\frac{\widehat{\tau}}{0}$ & 1 & 1 & $\stackrel{ }{=}$ & 1 & 1 & 1 & 1 \\
\hline- & 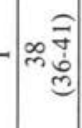 & $\stackrel{\stackrel{\infty}{=}}{=}$ & 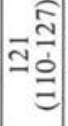 & œ & 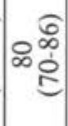 & 1 & 1 & 1 & 1 & $n$ & 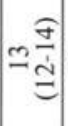 & $=\frac{\widehat{O}}{0}$ & 1 & 1 & 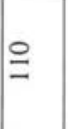 & 1 & 1 & 1 & 1 \\
\hline 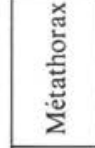 & 于啇 & $=\frac{\hat{D}}{\mathrm{I}}$ & 웜 & 今气 & 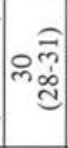 & ๙2 & m & $a \frac{6}{\infty}$ & 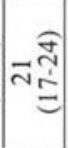 & 1 & $=\frac{\approx}{0}$ & $\stackrel{\frac{\sigma}{\sigma}}{\beth}$ & 。ำ & 0 & 1 & 1 & 1 & 1 & 1 \\
\hline 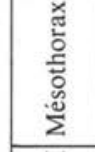 & 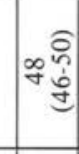 & 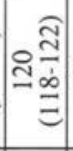 & 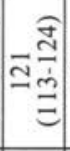 & $\stackrel{\infty}{\cong}$ & लॅ: & $2 \frac{2}{a}$ & 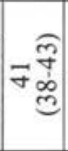 & $a \frac{6}{\infty}$ & 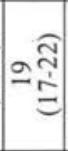 & 1 & $=\frac{\tilde{\sigma}}{0}$ & $2 \frac{\sigma}{\stackrel{\sigma}{\Xi}}$ & 올 & 옹 & 1 & 1 & 1 & 1 & 1 \\
\hline 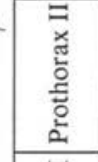 & 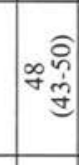 & $\underset{\infty}{\frac{\widehat{\Xi}}{\Xi}}$ & 옳 & गे & 1 & 응 & Ұ & $a \frac{\hat{0}}{\infty}$ & $\infty \frac{\sigma}{\tilde{s}}$ & 1 & 1 & 1 & 。 & of & 1 & 1 & 1 & 1 & 1 \\
\hline 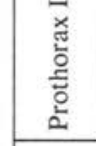 & $\infty \frac{\widehat{\sigma}}{\dot{0}}$ & 点 & సิ & 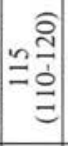 & ๙ิสิ & $\begin{aligned} & \stackrel{2}{0} \\
&= \frac{3}{3} \\
& \Xi\end{aligned}$ & 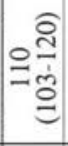 & 1 & 1 & 1 & in & 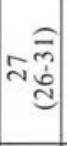 & 1 & 1 & 1 & 1 & 1 & 1 & 1 \\
\hline 芯 & 胥 & 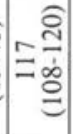 & 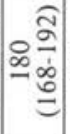 & 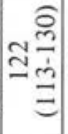 & 웛응 & $\therefore$ & 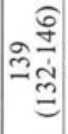 & ఇ & ڤั & 1 & 1 & 1 & 1 & 1 & 1 & 1 & 1 & 1 & 1 \\
\hline ڤั) & - & $N$ & $m$ & $\nabla$ & in & 0 & r & $\infty$ & $a$ & $\simeq$ & $\pi$ & مـ & 0 & $\tau$ & $<$ & $\infty$ & 0 & $\theta$ & (4) \\
\hline
\end{tabular}


2.2. Le thorax (fig. 6).

Les soies thoraciques présentent les mêmes caractères que chez $S$. ingrami. Leur morphologie et leur taille sont comparables. Les seules différences appréciables que nous ayons relevées concernent:

- la soie 1 du prothorax postérieur, du mésothorax et du métathorax, qui est un peu plus petite; courte.

- la microsoie $b$ du mésothorax et du métathorax, également barbulée, mais plus

\subsection{L'abdomen (fig. 6).}

Les soies abdominales, comme les soies céphaliques et thoraciques, sont très proches de celles de $S$. ingrami, avec une tendance générale à être légèrement plus petites. Les soies intersegmentaires suivent également cette tendance.

Dans cette région du corps, la seule différence nette se rapporte à la microsoie $a$, bien développée et barbulée chez $S$. ingrami, courte et pointue chez $S$. dureni.

\section{3. - La larve du $1^{\text {er }}$ Stade.}

Longueur totale: $0,74 \mathrm{~mm}(0,63-0,95)$;

Longueur des soies caudales : $0,84 \mathrm{~mm}(0,75-0,90)$;

Dimensions de la tête : $0,12 \mathrm{~mm}$ de longueur sur $0,10 \mathrm{~mm}$ de largeur.

Nous avons observé toutes les soies sans noter de différences importantes avec celles de la larve du $1^{\text {er }}$ stade de $S$. ingrami.

\section{4. - LA NYMPHE.}

L'observation de 19 exuvies nymphales n'a pas révélé de caractères spécifiques.

\section{Sergentomyia hamoni.}

\section{1. - L'đuf (fig. 7).}

Il mesure en moyenne $0,33 \mathrm{~mm}$ de longueur sur $0,11 \mathrm{~mm}$ de largeur. Son ornementation est également faite de granulations dessinant des polygones irréguliers.

2. - La larve du $4^{e} \operatorname{stade}$ ( fig. $8, A$ et $B$ et tableau III).

Les dimensions moyennes des 10 larves étudiées sont les suivantes:

- longueur totale : $2,54 \mathrm{~mm}(2,28-2,62)$;

- dimensions de la tête: $0,40 \mathrm{~mm}(0,39-0,42)$ de longueur sur $0,29 \mathrm{~mm}$ $(0,26-0,30)$ de largeur ; 


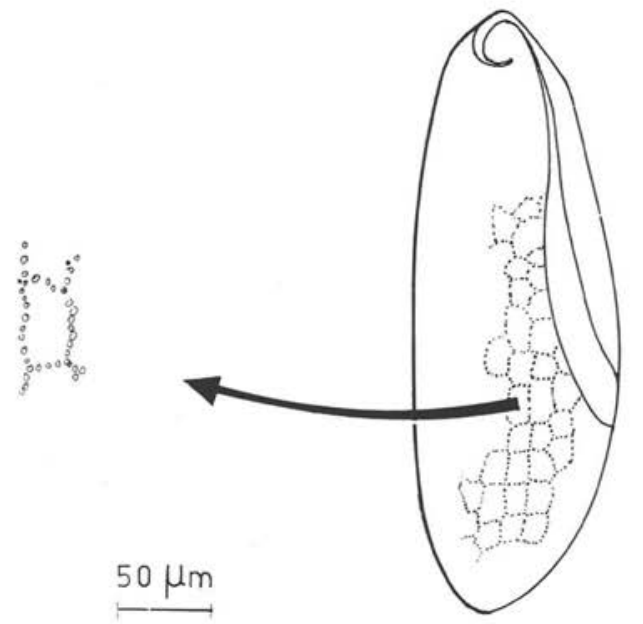

Fig. 7. L'œuf de S. hamoni.

- longueur des soies caudales internes : 2,02 mm (1,85-2,24);

- longueur des soies caudales externes : $1,81 \mathrm{~mm}(1,63-2,02)$.

Les soies de cette espèce présentent des particularités que nous n'avons pas encore rencontrées, aussi seront-elles décrites en détails.

\subsection{La tête (fig. 9).}

Comme chez les larves précédemment étudiées, les soies 1, 2, 4, 5, 6 sont lisses et les soies 7, 8, 9 épineuses. Seule distinction, les soies 3 sont plus densément barbulées, en particulier à leur extrémité.

\subsection{Le thorux.}

Prothorax antérieur (fig. 9).

Par leur structure et leurs proportions respectives, et bien que de tailles plus réduites, les soies de cette région rappellent celles de $S$. ingrami et $S$. dureni. Cependant les soies 2 présentent une morphologie particulière : elles ont l'aspect de fleurs à court pédoncule, à pétales d'égale longueur, disposés en verticilles réguliers; elles évoquent des fleurs de Nénuphars plus ou moins épanouies. A notre connaissance, de telles soies «anthomorphes» n'ont été observées jusqu'alors que chez $S$. hamoni.

Prothorax postérieur (fig. 9).

Soie 1 « anthomorphe». Soie 4 très réduite. Soie 2 bien développée $(81 \mu \mathrm{m})$.

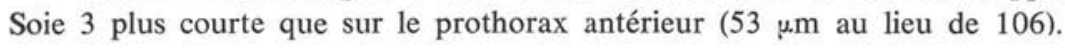


STADES PRE-IMAGINAUX DE PHLEBOTOMES

367
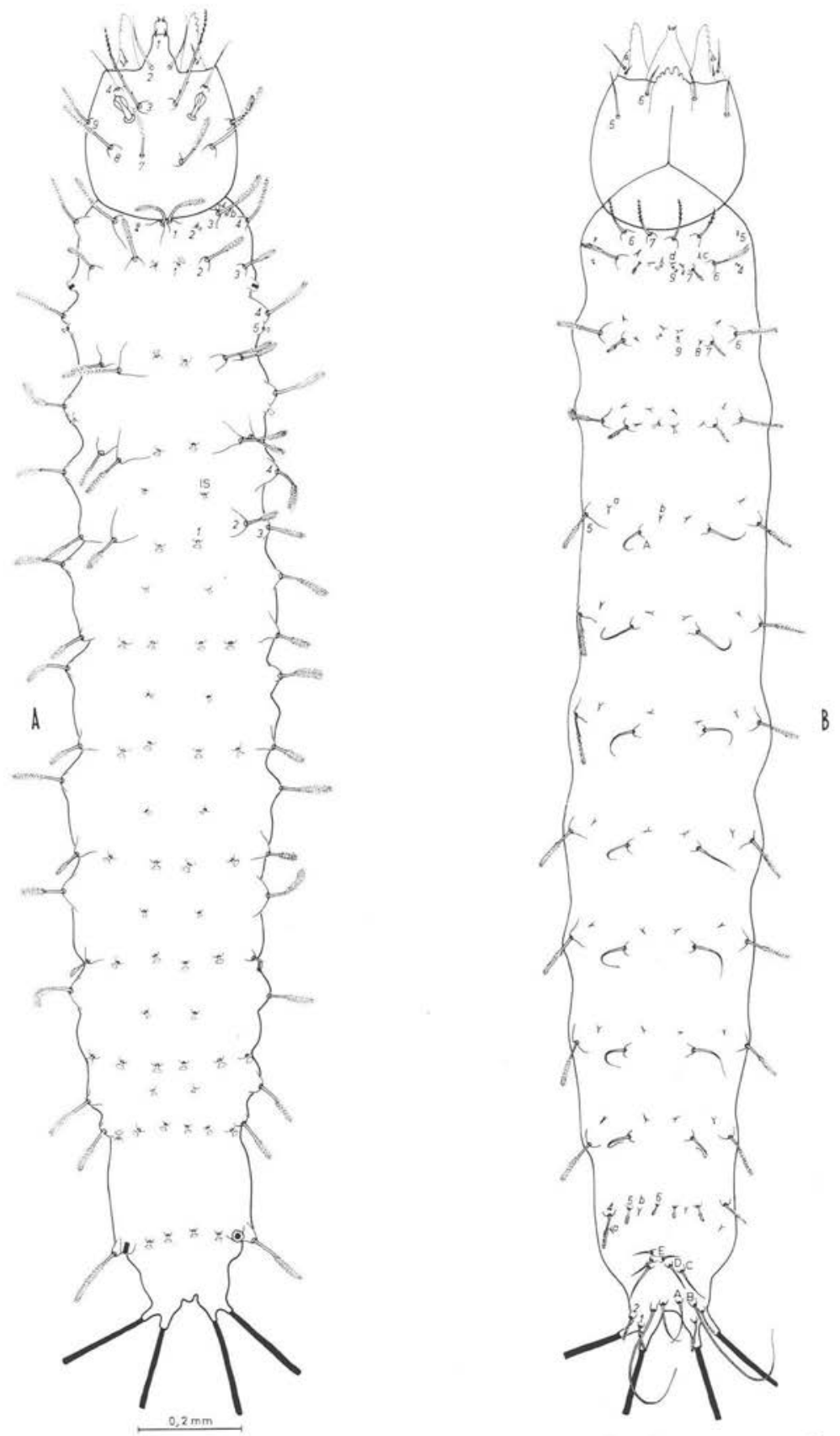

Fig. 8. S. hamoni. Larve du $4^{e}$ stade. A : vue dorsale; B: vue ventrale. 


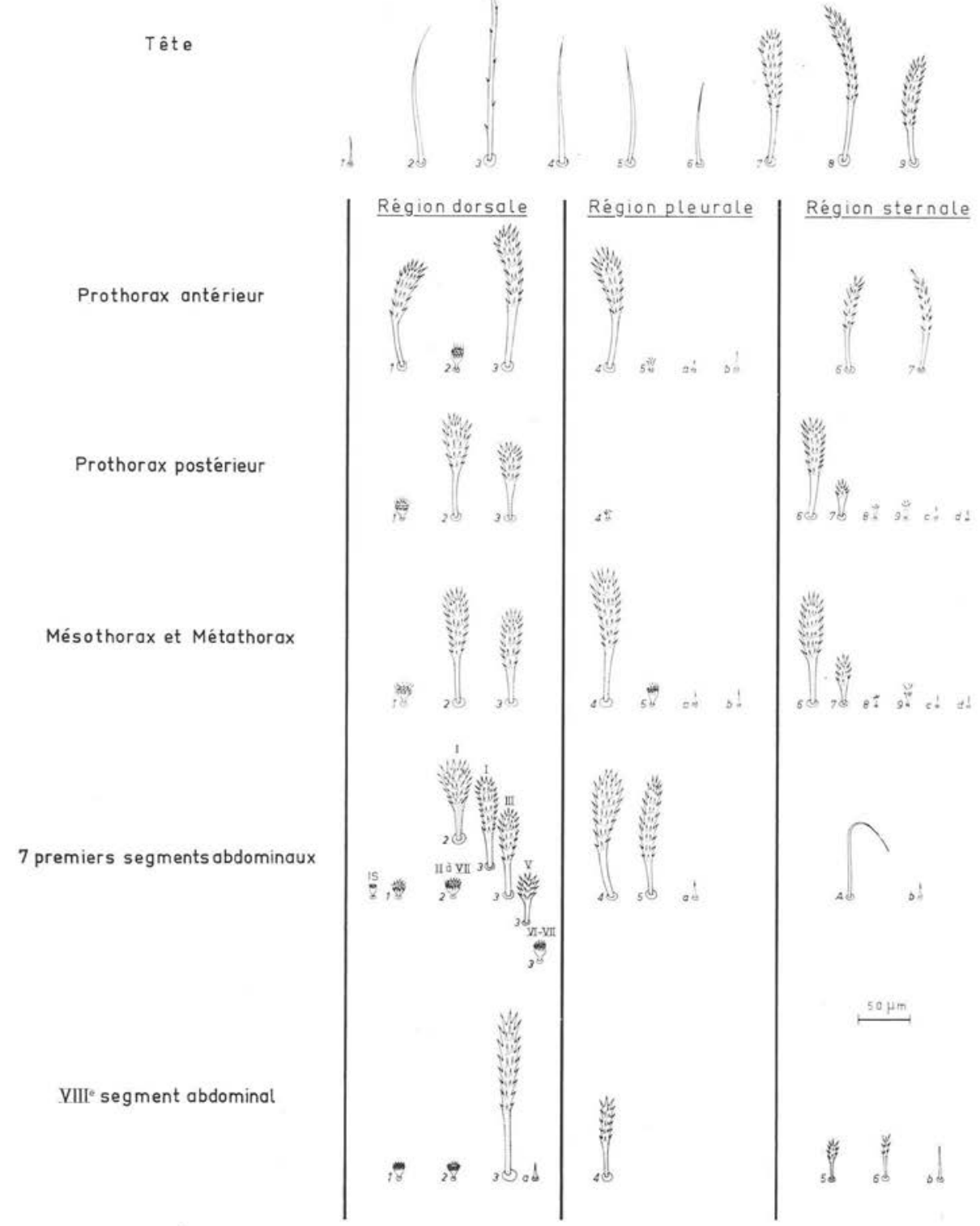

Fig. 9. S. hamoni. Soies de la larve du $4^{e}$ stade. 


\section{Mésothorax et métathorax (fig 9).}

Soies 1 et 5 «anthomoiphes». Soies 2, 3, 6, 7 comme sur le prothorax postérieur mais plus grandes. Soie 4 très développée. Soies 7 et 9 comparables en forme et en dimensions à leurs homologues du prothorax postérieur.

\subsection{L'abdomen.}

Les sept premiers segments abdominaux (fig. 9).

Soie 1 « anthomorphe» sur tous ces segments. Soie 2 développée $(59 \mu \mathrm{m})$ sur ie $I^{\text {er }}$ segment abdominal, " anthomorphe » sur les six autres. Soie 3 se réduisant progressivement du $\mathrm{I}^{\text {er }}$ au $\mathrm{V}^{\text {e }}$ segment, "anthomorphe»sur les segments VI et VII. Soies 4 et 5 bien développées sur tous les segments (respectivement 93 et $85 \mu \mathrm{m}$ ).

Les soies intersegmentaires sont égalemert "anthomorphes " avec environ 10 épines périphériques et 5 épines centrales.

Le VIII e segment abdominal (fig. 9).

Soies 1 et 2 «anthomorphes». Soies 3 très développée $(110 \mu \mathrm{m})$. Soies $4,5,6$ mesurant respectivement 62,28 et $27 \mu \mathrm{m}$.

Le IX segment abdominal.

Soies 1 et 2 épineuses. Soies A très largement barbulées. Soies B, C, D, E lisses.

3. - LA LARVE DU $1^{\text {er }}$ STADE.

Longueur totale : $0,49 \mathrm{~mm}(0,45-0,55)$;

Longueur des soies caudales: $0,76 \mathrm{~mm}(0,73-0,80)$;

Dimensions de la tête: $0,12 \mathrm{~mm}$ de longueur sur $0,08 \mathrm{~mm}$ de largeur.

3.1. La tête.

Soies comparables à celles de $S$. ingrami et $S$. dureni.

\subsection{Le thorax.}

Prothorax antérieur. Soies comparables à celles de S. ingrami et S. dureni.

Prothorax postérieur, mésothorax et métathorax. Les soies 1, 2, 3 sont épineuses mais nettement plus petites que chez $S$. ingrami et $S$. dureni. Les autres soies se présentent comme leurs homologues des deux autres espèces.

\subsection{L'abdomen.}

Sept premiers segments abdominaux. Comme sur le prothorax postérieur, le mésothorax et le métathorax, les soies 1, 2, 3 sont épineuses mais nettement moins développées que chez $S$. ingrami et $S$. dureni.

$V I I I^{e}$ et $I X^{e}$ segments abdominaux. Aucune différence importante n'a été notée par rapport aux soies de ces mêmes segments chez $S$. ingrami et $S$. dureni. 


\begin{tabular}{|c|c|c|c|c|c|c|c|c|c|c|c|c|c|c|c|c|c|c|c|}
\hline$\underline{x}$ & 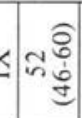 & 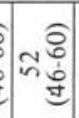 & I & 1 & 1 & 1 & 1 & 1 & & 1 & 1 & 1 & 1 & 1 & $\approx \frac{\hat{a}}{a}$ & 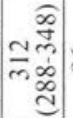 & 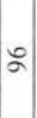 & & \\
\hline$\equiv$ & $=\frac{\widehat{\simeq}}{\varrho}$ & $=\frac{\widehat{A}}{0}$ & 웜 & 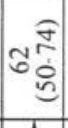 & 点 & ते & 1 & 1 & & 1 & $\sigma \frac{\widehat{T}}{5}$ & m气 & 1 & 1 & 1 & 1 & 1 & 1 & 1 \\
\hline 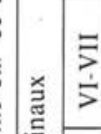 & $=\frac{\widehat{x}}{0}$ & $=\frac{\widehat{a}}{0}$ & $=\frac{\widehat{O}}{0}$ & 1 & 4 & 1 & 1 & 1 & & $0 \hat{v}$ & \pm & $\infty \widehat{\hat{L}}$ & 1 & 1 & $\cong$ & 1 & 1 & 1 & \\
\hline$>$ & $=\frac{\widehat{̃}}{0}$ & $=\frac{\widehat{x}}{0}$ & mîิ & & & 1 & 1 & 1 & & 0. & $\Xi$ & $\infty \stackrel{\hat{\underline{1}}}{=}$ & 1 & 1 & $\cong$ & 1 & 1 & 1 & \\
\hline ڤँ & $==\frac{\widehat{O}}{0}$ & $=\frac{\widehat{A}}{0}$ & 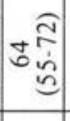 & 2 $\frac{\infty}{2}$ & œ & 1 & 1 & 1 & & $0 \stackrel{0}{0}$ & $\Xi$ & $\infty \stackrel{\widehat{a}}{5}$ & 1 & 1 & $\stackrel{2}{\beth}$ & 1 & 1 & 1 & \\
\hline$=$ & $==\frac{\widehat{\tau}}{0}$ & $=\frac{\widehat{A}}{0}$ & 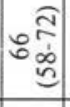 & & & 1 & 1 & 1 & & $0 \stackrel{0}{0}$ & $\Xi$ & $\infty \stackrel{\hat{\sigma}}{=}$ & 1 & 1 & $\cong$ & 1 & 1 & 1 & \\
\hline - & 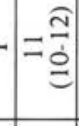 & 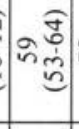 & 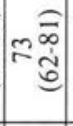 & $Y$ & $\nabla$ & 1 & 1 & 1 & & $0 \stackrel{5}{6}$ & $\Xi$ & $\infty \stackrel{\hat{\grave{2}}}{=}$ & 1 & 1 & $\cong$ & 1 & 1 & 1 & 1 \\
\hline 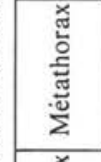 & $=\frac{\widehat{\tau}}{0}$ & $\approx \frac{\infty}{a}$ & م & 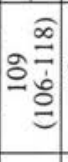 & 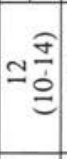 & œ & ले & $\circ \frac{\bar{a}}{a}$ & $\stackrel{\widetilde{N}}{-\underset{\infty}{=}}$ & 1 & $=\frac{\tilde{a}}{0}$ & 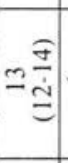 & $0 \stackrel{0}{i}$ & $0 \stackrel{0}{6}$ & I & 1 & 1 & 1 & I \\
\hline 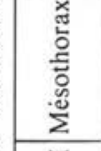 & $=\frac{\widehat{T}}{0}$ & $\therefore \frac{0}{b}$ & 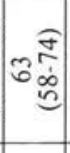 & ก응 & $\simeq \stackrel{\vartheta}{\Xi}$ & $\infty \frac{8}{\circ}$ & \% & $\circ=$ & $\begin{array}{l}\widehat{\widehat{\pi}} \\
-\underset{\infty}{=}\end{array}$ & 1 & $=\frac{\widehat{\partial}}{\underline{0}}$ & 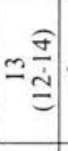 & 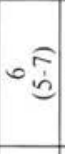 & $0 \stackrel{0}{0}$ & 1 & 1 & 1 & 1 & 1 \\
\hline 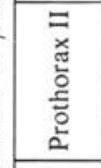 & $\cong \stackrel{\widehat{\theta}}{\Xi}$ & 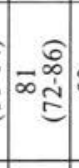 & 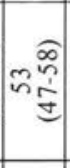 & 음 & 1 & ה & حૅ & 음 & $0 \frac{E}{\frac{E}{\sigma}}$ & 1 & 1 & 1 & o $0 \stackrel{5}{6}$ & 욜 & I & 1 & 1 & 1 & 1 \\
\hline 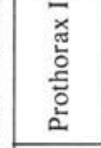 & 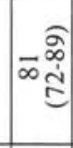 & $\pm \underset{\Xi}{\stackrel{\Xi}{\Xi}}$ & ๕๕ & ๙2. & $\simeq \stackrel{\widehat{\Im}}{0}$ & 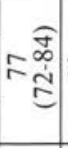 & 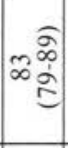 & 1 & 1 & 1 & in & 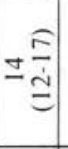 & 1 & 1 & 1 & 1 & 1 & 1 & 1 \\
\hline & సสำ & $\stackrel{\circ}{\circ}$ & \begin{tabular}{r}
$\underset{\infty}{\infty}$ \\
$\approx$ \\
\hdashline$\underset{\infty}{\infty}$ \\
$\Xi$
\end{tabular} & 등 & $\infty \frac{\widehat{\sigma}}{\grave{\infty}}$ & 동 & 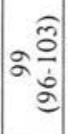 & 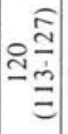 & 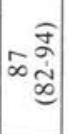 & I & 1 & 1 & 1 & 1 & 1 & 1 & 1 & 1 & 1 \\
\hline ڤั) & - & $\sim$ & $m$ & $\nabla$ & n & 6 & $r$ & $\infty$ & $\sigma$ & $\cong$ & ז & م & 0 & $\nabla$ & $<$ & $\infty$ & $u$ & 0 & (피 \\
\hline
\end{tabular}


4. LA NYMPHE.

Les 9 exuvies nymphales examinées, comme celles de $S$. ingrami et $S$. dureni, ne présentent pas de caractères particuliers.

\section{III. - Notes bio-écologiques}

\section{1. Cycle évolutif.}

Les femelles gorgées ont respectivement pondu: la femelle de S. ingrami : 130 œufs, les deux femelles de $S$. dureni : 94 et 96 œufs, les vingt-cinq femelles de $S$. hamoni: 75 œufs en moyenne (24-115).

L'insectarium utilisé, installé en sous-sol, présente des conditions relativement constantes qui sont, en saison des pluies: température $26^{\circ} \mathrm{C}$, hygrométrie : 88 à $96 \%$, obscurité presque complète.

Pour ces trois espèces, les durées de développement post-embryonnaire ont été les suivantes :

\begin{tabular}{|c|c|c|c|}
\hline \multirow{2}{*}{ Stade } & \multicolumn{3}{|c|}{ Espèce } \\
\hline & S. ingrami* & S. dureni* & S. hamoni* \\
\hline 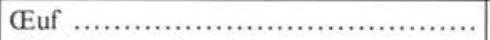 & $8 \mathrm{j}$. & $5 \mathrm{j}$. & $9 \mathrm{j}$. \\
\hline 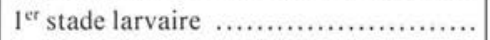 & $6 \mathrm{j}$. & $6 \mathrm{j}$. & $5 \mathrm{j}$. \\
\hline $2^{\mathrm{c}}$ stade larvaire $\ldots \ldots \ldots \ldots \ldots \ldots \ldots \ldots \ldots \ldots \ldots \ldots \ldots \ldots \ldots \ldots$ & $3 \mathrm{j}$. & $3 \mathrm{j}$. & $3 \mathrm{j}$. \\
\hline 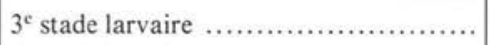 & $4 \mathrm{j}$. & $4 \mathrm{j}$. & $3 \mathrm{j}$. \\
\hline $4^{e}$ stade larvaire $\ldots \ldots \ldots \ldots \ldots \ldots \ldots \ldots \ldots \ldots \ldots \ldots \ldots \ldots \ldots \ldots \ldots$ & $8 \mathrm{j}$. & $5 \mathrm{j}$. & $6 \mathrm{j}$. \\
\hline 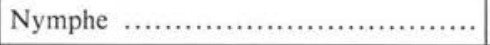 & $9 \mathrm{j}$. & $9 \mathrm{j}$. & $6 \mathrm{j}$. \\
\hline Durèe du cycle évolutif ................... & 38 j. -42 j.** & $32 \mathrm{j} .-41$ j.** & $32 \mathrm{j} .-52 \mathrm{j} . * *$ \\
\hline
\end{tabular}

* Pour chaque stade est portée la durée minimale obtenue en observant la première apparition de ce stade.

** Durée maximale obtenue en observant la dernière émergence.

En ce qui concerne $S$. hamoni, ne sont considérées ici que les pontes qui ont connu un développement régulier. En effet, une diapause ou quiescence observée au stade œuf, fait actuellement l'objet d'une étude.

\section{2. Ecologie.}

Deux de ces trois espèces, $S$. ingrami et $S$. hamoni, sont essentiellement forestières. Par contre, $S$. dureni est un Phlébotome de savane, fréquent dans les villages où, attiré par la lumière, il pénètre dans les habitations (Vattier-Bernard et Trouillet, 1978). 


\section{Discussion}

Les larves de $S$. ingrami et $S$. dureni présentent une chétotaxie très voisine dont les caractères principaux sont:

— sur le prothorax antérieur: soie 2 réduite;

- sur le prothorax postérieur, le mésothorax et le métathorax : soie 1 nettement plus petite que les soies 2 et 3 ;

- sur les sept premiers segments abdominaux: seules les soies 3 ont une taille constante, les soies 1 et 2 décroissent régulièrement du $\mathrm{I}^{\text {er }}$ au $\mathrm{VII}^{\circ}$ segment ;

- sur le $\mathrm{VIII}^{\circ}$ segment abdominal : soie 1 réduite, soies 2 et 3 bien développées.

Nous sommes donc amené à constater une similitude de la chétotaxie chez ces deux espèces du sous-genre Rondanomyia. Seule l'étude des larves de nombreuses espèces pourra faire découvrir si cette règle est générale.

Les larves de $S$. hamoni montrent de nombreuses particularités qui permettent de les distinguer facilement des précédentes:

- soie 3 céphalique à extrémité distale très barbulèe ;

- soie 1 « anthomorphe », du prothorax postérieur au VIII ${ }^{e}$ segment abdominal ;

- soie 2 assez développée sur le prothorax postérieur, le mésothorax, le métathorax et le $\mathrm{I}^{\text {er }}$ segment abdominal, et «anthomorphe» du $\mathrm{II}^{\mathrm{e}}$ au VIII ${ }^{\mathrm{e}}$ segment abdominal ;

- soie 3 assez développée sur le prothorax postérieur, le mésothorax, le métathorax, le $\mathrm{I}^{\text {er }}$ segment abdominal. Elle se réduit progressivement du $\mathrm{I}^{\mathrm{er}}$ au $\mathrm{V}^{\mathrm{e}}$ segment abdominal, devient «anthomorphe» sur le $\mathrm{VI}^{e}$ et le $\mathrm{VII}^{\mathrm{e}}$, est à nouveau bien développée sur le VIII".

- $\mathrm{VIII}^{\mathrm{e}}$ segment abdominal avec soies 1 et 2 réduites, soie 3 bien développée. Chez d'autres espèces (Grassomyia ghesquierei, S. bedfordi firmatus, S. bedfordi medius, $S$. ingrami, $S$. dureni), le VIII ${ }^{\mathrm{e}}$ segment abdominal présente une soie 1 réduite, les soies 2 et 3 étant bien développées (Trouillet, 1976 et 1977).

\section{Bibliographie}

Abonnenc E. (1956): L'œuf et les formes pré-imaginales de deux Phlébotomes africains $P$. antennatus var. occidentalis Theodor, 1933 et P. dubius Parrot, Mornet et Cadenat, 1945. Arch. Inst. Pasteur Alger, 34, 518-539.

Abonnenc E. (1972): Les Phlébotomes de la région éthiopienne (Diptera, Psychodidae). Mém. O.R.S.T.O.M., 55, 289 p.

Gemetchu T. (1971): Liver and yeast as larval diets in colonization of a sandfly (Phlebotomus longipes). Trans. R. Soc. Trop. Med. Hyg., 67, 434. 
Killick-Kendrick R., Leaney A. J., Ready P. D. (1977) : The establishment, maintenance and productivity of a laboratory colony of Lutzomyia longipalpis (Diptera: Psychodidae). J. Med. Ent., 13, $429-440$.

Trouillet J. (1976) : Sergentomyia (Grassomyia) ghesquierei Parrot, 1929 (Diptera, Phlebotomidae). Etude morphologique des stades pré-imaginaux et notes bio-écologiques. Cah. O.R.S.T.O.M., sér. Ent. Méd. Parasitol., 14, 347-356.

Trouillet J. (1977) : Sergentomyia (Sergentomyia) bedfordi firmatus Parrot et Malbrant, 1945 et Sergentomyia (Sergentomyia) bedfordi medius Kirk et Lewis, 1950 (Diptera, Phlebotomidae). Etude morphologique des stades pré-imaginaux et notes bio-écologiques. Ann. Parasitol. Hum. Comp., 52, 35-52.

Trouillet J., Makany L., Cros B., Grillot J.-P.: Recherches bio-écologiques dans la région de Yaka-Yaka (Congo). 1. Présentation du milieu. Ann. Univ. Brazzaville (sous presse).

Vattier-Bernard G. et Trouillet J. (1978) : Les Phlébotomes (Diptera, Phlebotomidae) du Mayombe (République Populaire du Congo). Ann. Parasitol. Hum. Comp., 53, 697-704. 